\title{
Traduire
}

Une autre perspective sur r tr traduction

Revue française de la traduction

$220 \mid 2009$

Organisations internationales | Bicentenaire de Louis Braille

\section{Dire presque la même chose - Expériences de traduction, Umberto Eco}

Maurice Voituriez

\section{(2) OpenEdition \\ Journals}

Édition électronique

URL : http://journals.openedition.org/traduire/399

DOI : $10.4000 /$ traduire.399

ISSN : 2272-9992

Éditeur

Société française des traducteurs

Édition imprimée

Date de publication : 15 juin 2009

Pagination : 73-74

ISSN : 0395-773X

Référence électronique

Maurice Voituriez, "Dire presque la même chose - Expériences de traduction, Umberto Eco », Traduire [En ligne], 220 | 2009, mis en ligne le 12 novembre 2013, consulté le 22 septembre 2020. URL : http:// journals.openedition.org/traduire/399 ; DOI : https://doi.org/10.4000/traduire.399 


\section{Compte-rendu d'ouvrage \\ "PRESQUE ... "}

\section{Maurice Voituriez}

Bien souvent - et encore récemment - nous regrettions que tant de livres consacrés à notre métier contiennent si peu d'exemples concrets. L'ouvrage d'Umberto Eco intitulé en français Dire presque la même chose - Expériences de traduction nous apporte, à cet égard, un puissant, et double, réconfort. Puissant par l'abondance des exemples et double par la place dominante accordée à la langue de Dante. Cela n'est pas surprenant puisque cet auteur est Italien mais c'est bienvenu. Comme on dit, cela nous change ! Écrivain célèbre, ex-professeur (à Paris), manifestement polyglotte et lui-même traducteur, Eco était vraiment prédestiné à écrire sur ce sujet. Traduit lui-même en de nombreuses langues, il a l'expérience irremplaçable des contacts personnels - et certainement ardus - avec ses traducteurs. On sait en effet combien peuvent être difficiles les relations entre n'importe quel de nos confrères et un auteur qui connaît les deux langues et serait presque apte à se traduire lui-même !

Eco fait allusion à ce genre de problèmes et illustre son propos en présentant des textes à partir de l'italien et vers le français, l'anglais, l'espagnol, le néerlandais (hélas, improprement dénommé : hollandais !) et même le latin. Quelquefois aussi en sens inverse ! N'oublions pas qu'il a notamment traduit Nerval et Raymond Queneau.

L'éditeur assure que l'ouvrage " réjouira " traducteurs, enseignants et étudiants. C'est bien vrai, en tout cas, pour nos confrères et, en outre, il s'agit d'un outil didactique précieux, particulièrement pour les italianisants de tous niveaux. Mais donnons la parole à Eco :

Je me demande si, pour élaborer une théorie de la traduction, il ne serait pas nécessaire d'examiner de nombreux exemples de traduction, mais aussi d'avoir fait trois expériences : avoir vérifié les traductions d'autrui, avoir traduit et été traduit, ou mieux encore, avoir été traduit en collaboration avec son traducteur.

Le bon apôtre ! II a parfaitement conscience d'être une des rares personnes à remplir, dans le domaine littéraire, l'ensemble de ces conditions !

Redonnons-lui cependant la parole puisqu'il rend hommage à notre profession, ce qui n'est pas le cas de tous les écrivains : 
En général, ce n'est pas tant l'auteur qui influence le traducteur, mais plutôt le traducteur qui, demandant un soutien à l'auteur pour une modification qu'il sait hardie, lui permet de comparer le véritable sens de ce que lui, l'auteur avait écrit.

Nous avons plaisir à citer Eco mais que le lecteur sache bien qu'il s'efface devant une multitude d'exemples, qui constituent l'essentiel du livre et ici ce n'est pas l'anglais qui prédomine.

II n'est pourtant pas absent - pourquoi le serait-il ? - et certains termes comme l'anglais américain downtown et uptown, apparemment simples, ne le sont pas tellement à ses yeux : Downtown est certes le quartier des affaires, mais aussi celui du vice (" les bas quartiers").

L'auteur en conclut " qu'un traducteur doit connaître la langue, mais aussi la topographie de chaque ville ". En l'occurrence, de la ville américaine concernée.

En page 364 il cite James Joyce et mentionne, par exemple, en italien la rivière Schelda, qu'il ne parvient pas à situer. II s'agit de l'Escaut (Schelde en néerlandais).

Mais - nous l'avons déjà dit - ce sont les langues latines qui occupent la place d'honneur. Dans le cas de la Sylvie de Nerval, Eco explique ses problèmes de passage à l'italien, notamment dans la description du vêtement féminin, pas si simple pour un confrère du xxe siècle.

II traite aussi de l'" allusion ", élément quelquefois essentiel de la traduction littéraire et faisant appel à la culture générale, denrée assez rare par le temps qui court !

De tout ce qui précède on pourrait conclure, parodiant Beaumarchais : "Aux qualités qu'on attend d'un traducteur, combien d'écrivains seraient-ils aptes à passer un bac-langues ? "

La perfection n'étant pas de ce monde, on peut regretter une certaine lourdeur de style et trop de jargon. C'est un peu malheureusement la loi du genre et les productions d'Eco n'y échappent guère... surtout si elles sont elles-mêmes traduites ! Il y a même quelques fautes de ponctuation et de syntaxe, ce qui est dommage.

Qu'Umberto Eco ait profité de l'occasion pour citer largement ses propres œuvres, cela ne nous choque pas tellement de la part d'un écrivain renommé. La tentation était trop forte!

En tout cas, voilà un livre presque unique en son genre, sortant des chemins battus de la traductologie. Rien que pour cela, grâce lui soit rendue ! Puisse-t-il susciter d'autres vocations !

mvoituriez@wanadoo.fr

Dire presque la même chose - Expériences de traduction, Umberto ECO, Éditions Grasset et Fasquelle, Paris, 2006, Traduit de l'italien par Myriem Bouzaher. 460 pages. 